\title{
Parity violation in chiral structure creation under femtosecond laser irradiation in silica glass?
}

\author{
Bertrand Poumellec ${ }^{1}$, Matthieu Lancry ${ }^{1}$, Rudy Desmarchelier ${ }^{1}$, Evelyne Hervé ${ }^{2}$ and Bernard Bourguignon ${ }^{3}$ \\ The paper addresses the creation of circular optical properties from a femtosecond laser light beam with a linear polarization in \\ an achiral material (glass) under an orthogonal incidence. In this situation, all aspects of the experiment are achiral and \\ therefore should not give rise to chiral property creation. From that observation, we propose an interpretation that involves the \\ action of a light-induced torque on the matter carrying a light-induced dielectric moment. We found that a direct current (DC) \\ electric field could be produced in the lattice by the femtosecond laser in our conditions and that a non-collinear dielectric \\ moment is created by a nonlinear effect between the DC electric field and the stress field due to the transformation of the mate- \\ rial. We reveal that it is possible to break the chiral symmetry of glass using an intense, ultrashort laser light pulse.
} Light: Science \& Applications (2016) 5, e16178; doi:10.1038/lsa.2016.178; published online 18 November 2016

Keywords: chirality; chiroptical properties; circular dichroism; femtosecond laser processing; glass; optical rotation; silica

\section{INTRODUCTION}

Femtosecond laser pulses focused in silica are absorbed through nonlinear photoionization mechanisms; such mechanisms induce various modifications in the glass volume, depending on the laser parameters, such as refractive index changes ${ }^{1}$, the formation of threedimensional (3D) nanostructures ${ }^{2}$, or voids ${ }^{3}$. Applications are numerous in a large number of domains, including health ${ }^{4}$, electronics, optical data storage ${ }^{5-7}$, optofluidics ${ }^{8,9}$ and optical components (for example, 3D optical waveguides ${ }^{1,10}$ or polarizing optical devices $)^{11-14}$. Considerable research efforts are underway in the laser processing of optical materials because it is possible to achieve strong refractive index increase (a few $10^{-3}$ to $>10^{-2}$ in a large range of transparent glasses) localized in the bulk (due to a strong nonlinear effect) and because the spatial resolution coincides with the requirement for waveguide elaboration (a few $\mu \mathrm{m}$ ). It is thus necessary to understand how to control the interaction of femtosecond laser light with optical materials, in particular, with optical glasses, the most important of which being silica. If one proves to be able to control the forces involved in these processes, one could exceed the current applications of the lasers and open new possibilities in materials sciences.

In the past decade, several new phenomena have been discovered: nanogratings for high birefringence application with polarization direction dependence ${ }^{15}$, 'quill' writing ${ }^{16}$ with dependence of modification with the direction of writing, silica decomposition ${ }^{17}$, shear stressinduced field ${ }^{18}$ and an efficient creation of oriented anisotropic defects ${ }^{19}$. All these findings show that asymmetric printing in a centrosymmetric medium could be controlled ${ }^{20}$. This concept of symmetry breaking (the Pierre Curie principle stipulates that the symmetry of the effects is always higher than the symmetry of the causes ${ }^{21}$ ) from nonlinear interaction of the infrared (IR) light appeared with the development of nonlinear optics and optical fibers. A famous example is the spontaneous appearance of second harmonic generation under IR irradiation ${ }^{22}$, which is normally forbidden in centrosymmetric media. Alternatively, it is conceivable that chiral light action in an achiral material can produce chiral modification. However, the efficiency is usually low or undetectable if an amplification process is not used ${ }^{23}$.

In 2003, our group proved that femtosecond laser interaction can shear matter in a manner similar to a scissor cutting material, moving matter on one side in one direction and matter on other side in the opposite direction, depending on the laser scanning direction and on the femtosecond light polarization ${ }^{18}$. It was surprising to discover that an achiral beam (axially symmetric distribution of intensity, linearly polarized) penetrating into an achiral material (namely, pure silica glass) in an achiral geometry (orthogonal incidence) can give rise to a chiral strain if we take into account the light propagation axis. This observation was performed in the domain of the strain field (especially regarding shearing) and this strain does not give rise to optical circular properties by itself like the torsion can do in matter. However, in the present paper, it is demonstrated that another phenomenon occurring in the laser-matter interaction can actually lead to imprinting of chiral properties within a glass material. This reveals a parity violation (the reciprocity of the light propagation in the glass is broken) by efficient chiral structure creation. This chiral structure leads to the introduction of a chiral force (similar to the creation of torque) with a tightly focused femtosecond laser light in transparent solid material via the combination of nonlinear effects.

${ }^{1}$ SP2M/ICMMO, CNRS-UPSud, Université Paris Sud, Université Paris Saclay, Bâtiment 410, Orsay 91405 Cedex, France; ${ }^{2}$ Centre des matériaux, Mines Paris, Paristech, UMR CNRS-UPMC 7633, BP87, Evry F-91003 Cedex, France and ${ }^{3}$ ISMO, CNRS-UPSud, Université Paris Sud, Université Paris Saclay, Bâtiment 210 , Orsay 91405 Cedex, France Correspondence: B Poumellec, Email: bertrand.poumellec@u-psud.fr

Received 28 June 2015; revised 27 May 2016; accepted 7 June 2016; accepted article preview online 8 June 2016 
This new technology enables the creation and the control of the circular properties, namely, rotating power and circular dichroism (CD). Currently, it is not possible to obtain similar properties in solids other than in elaborating single crystals. Such optical activity has a crucial role in optoelectronic devices, biological sensing and analytical chemistry. This new technology could provide a platform for a versatile new class of photonic devices. We can imagine a switchable plasmonic material based on chiral plasmonic nanostructures on glass surfaces (for example, DNA nanostructures decorated with metal nanoparticles) and a plethora of chiral photonic devices. More generally, this approach paves the way towards control of the orientation of the crystallization of the compound of interest, for example, the materials for second harmonic generation or for piezoelectric or ferroelectric effects, that is, second-order nonlinear materials.

\section{MATERIALS AND METHODS}

Femtosecond laser writing

The laser radiation, in a Gaussian mode, was produced by a regenerative amplified mode-locked Ti:sapphire laser operating at $100 \mathrm{kHz}$ repetition rate that produces 160 -fs pulses of light at a wavelength of $800 \mathrm{~nm}$ or by a femtosecond laser system (Satsuma, Amplitude Systèmes Ltd, Allée de Canteranne, Pessac, France) operating at $100 \mathrm{~s}$ of $\mathrm{kHz}$ and delivering pulses of $300 \mathrm{fs}$ in duration at a wavelength of $1030 \mathrm{~nm}$. Considering that the propagation vector $\vec{k}$ is along the $\vec{z}$ direction, the beam was focused from 100 to $200 \mu \mathrm{m}$ below the entry surface using a 0.5 numerical aperture (NA) microscope objective. The writing configurations defined by writing and polarization directions are shown in Figure 1. Therefore, when the laser was moving along $X$ (or $Y$ ) and the polarization was lying along $x$, it is defined as ' $X x$ writing' (or ' $Y x$ writing'). Next, moving the sample along the $X$ or $Y$ axis at a scanning speed of $500 \mu \mathrm{m} \mathrm{s}^{-1}$, either single lines or $10 \mathrm{~mm}$ squares made of a group of lines were photoinduced in silica plates with various pulse energies ranging within a few fractions of $\mu \mathrm{J}$ per pulse and at different polarization directions (along the $X$ or $Y$ axis). The lines were written while paying attention to their direction (referenced to the laboratory) and orientation (for example, left to right and right to left). The irradiated squares were written following a linear scanning trajectory with a $1-\mu \mathrm{m}$ pitch to achieve uniformly scanned region and eliminate any diffraction effects. Subsequently, some single laser lines were observed in transmission using a polarized optical microscope for detecting neutral axes, determining the slow axis orientation, optical rotation power and for measuring the linear birefringence via the Sénarmont method. Square samples were used to perform the CD measurements using a JASCO J-810 spectro-polarimeter (Jasco Company, rue des cerisiers, Lisses, France) equipped with a $50 \mathrm{kHz}$ photoelastic modulator. Measurements were performed in the $185-700 \mathrm{~nm}$ spectral range with a 1-nm spectral resolution and with a 2 -s integration time, resulting in an error of $<1 \mathrm{mdeg}$.

Measurement of CD in a substance exhibiting heterogeneous linear dichroism in depth but with transverse homogeneity

Usually, CD is measured in homogeneous substances having a center of symmetry but no circular symmetry, as in the case of a liquid. $\mathrm{CD}$ is measured by recording the light attenuation through the sample alternating left and right circular polarization using a photoelastic modulator. The attenuation difference normalized by the sum is called the ellipticity $\theta$ (expressed in mdeg). The relation with the $\mathrm{CD}$ is $\theta=\left(\alpha_{\mathrm{L}}-\alpha_{\mathrm{R}}\right) e$, where $\alpha$ is the absorption and $e$ is the circular property thickness.

In addition, in the case of a sample mixing uniformly linear and circular properties in depth, the isotropy is broken and we can suspect a dependence of the properties on the azimuthal position of the sample. The calculation shows that such mixing is not the case and the angular position of the neutral axes of the sample can be ignored.

In the case of a sample exhibiting a non-uniform distribution of linear and circular properties, any series of rotator and dephasor matrices can be reduced to a simple combination of one rotator $(R)$ and one dephasor $(D)$ matrices in arbitrary order, containing the equivalent rotation angle, the equivalent slow axis angle and the equivalent dephasing angle. In addition, in the case of linear properties much larger than the circular one, the equivalent orientation of the slow axis of the equivalent dephasor can be ignored and set to
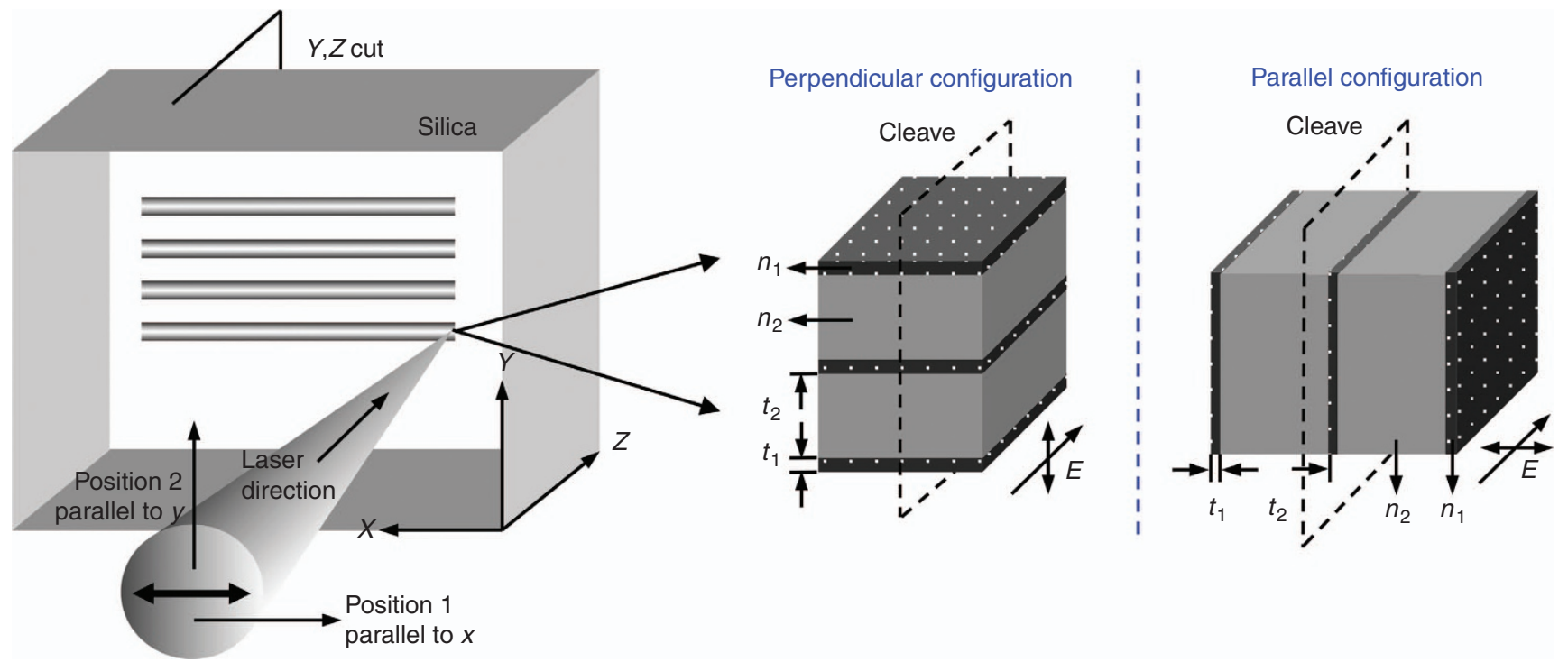

Figure 1 Experimental setup scheme of the configuration for writing (from the left side) and schematic of a sub-wavelength periodic structure formed in a cross-section of the irradiated region. $n_{1}$ and $n_{2}$ : local refractive indices of the nanoplanes; $t_{1}$ and $t_{2}$ are their respective thicknesses. Position 1 and 2 refer to light polarization. Reproduced from Desmarchelier et al. ${ }^{24}$, Copyright @ 2015 by Desmarchelier et al. and Scientific Research Publishing Inc. 
zero. It is thus possible to simulate the sample action as $R(\varphi) D(\phi)$ through one face and $D(\phi) R(\varphi)$ through the other face, arbitrarily. $\varphi$ is the complex equivalent circular angle containing the circular birefringence $(\operatorname{Im}(\varphi)$, imaginary part) and dichroism $(\operatorname{Re}(\varphi)$, real part); $\phi$ is the same for the linear properties. In this case, the ellipticity $\theta$ follows $\tanh (\theta)=\tanh (2 \operatorname{Im}(\varphi))$ when incident on one sample face and $\tanh (\theta)=\tanh [2 \operatorname{Im}(\varphi)] \cos [\operatorname{Re}(\phi)] / \cosh [\operatorname{Im}(\phi)]$ when incident on the other sample face. This result shows that the ellipticity difference between faces arises from the linear optical properties and that a non-zero ellipticity indicates the presence of $\mathrm{CD}$.

From a practical perspective, this result shows that the light propagation is likely to be non-reciprocal in our samples, and thus, ellipticity measurements must be performed from both sides of the sample. Fortunately, the measurement is still independent of the azimuthal rotation of the sample.

\section{RESULTS AND DISCUSSION}

\section{Femtosecond laser writing}

Usually, in pure silica, at low exposure energy $(E<0.31 \mu \mathrm{J}$ per pulse for laser polarization parallel to the scanning direction and $0.5 \mathrm{NA}$, $160 \mathrm{fs}$ ), one detects only a very weak linear birefringence of $<2 \times 10^{-4}$. The femtosecond laser interaction with silica is nearly 'isotropic' and is mainly due to the density changes that we attribute to an increase in the glass fictive temperature ${ }^{25}$. On the contrary, beyond the T2 threshold, a strong linear birefringence appears. Measured retardance is approximately a few hundreds of nanometers for an interaction length of a few 10's of $\mu \mathrm{m}$. Thus, this birefringence is on the order of $10^{-2}$, similar to quartz. This birefringence was shown by Kazansky et al. ${ }^{26}$ to be related to the formation of nanoplanes of low refractive index ${ }^{2}$. Recently, we established that nanoplanes are formed by the decomposition of $\mathrm{SiO}_{2}$ into nanoporous silica. In the following, most experiments were performed in the laser parameter domain of existence of porous nanoplanes domain.

\section{Rotated neutral axes}

For this specific observation, single lines of glass modifications, separated typically by $100 \mu \mathrm{m}$ at least, were written in glass plates, as described in the section Femtosecond laser writing. These lines were then placed under a polarized optical microscope for examination in transmission and birefringence quantitative measurements. The samples exhibited strong linear birefringence on the order of $250 \mathrm{~nm}$ for a thickness of $40 \mu \mathrm{m}$, that is, $6 \times 10^{-3}$. From the symmetry of the writing and when the polarization is aligned or perpendicular to the written line direction, it is expected that one neutral axis aligned with the written line direction and the other one aligned perpendicular to $i^{27}$. However, it was surprising to observe that complete optical extinction was not possible between crossed polarizers. It was necessary to slightly uncross the analyzer, that is, the neutral axis turned a few degrees around the laser propagation axis. This departure angle depends on the writing sense in such a way that it is possible to vary the contrast of the line written in one sense over the others written in the other sense just by rotating the sample between crossed polarizers. This observation leads us to consider the optical rotating power.

\section{Optical rotation}

One of the sample used in the section Rotated neutral axes was used for measuring the optical rotation. The maximal extinction between crossed polarizers was searched, and the analyzer was then uncrossed for tentatively improving the optical extinction. We found an uncrossing angle of $0.5^{\circ}$ for a $50 \mu \mathrm{m}$ (or less) active thickness corresponding to an optical rotation of $10^{\circ} \mathrm{mm}^{-1}$ at $632 \mathrm{~nm}$; such an optical rotation adequate for practical applications. For this sample, the irradiation parameters were as follows: $1.5 \mu \mathrm{J}, 1030 \mathrm{~nm}, 100 \mathrm{kHz}$, $0.6 \mathrm{NA}$ and $300 \mathrm{fs}$. Note that the rotating power for quartz is $\sim 21.73^{\circ} \mathrm{mm}^{-1}$ at $589 \mathrm{~nm}$ (Ref. 28). The sign is not defined because it is determined by the crystal arrangement, which can be levogyre or dextrogyre. No rotating power was detected for pulse energies below the porous nanoplanes formation ${ }^{29}$. This result is the first piece of evidence that the femtosecond laser can render a glass chiral at the macroscopic level. In solids, optical chirality can originate from chirality at the molecular level and/or from a chiral arrangement of achiral motifs, as in quartz itself (which is, by the way, a crystalline phase of silica) ${ }^{29}$. The irradiated glass is thus linearly and circularly birefringent. In that case, some additional CD can be expected.

\section{Spectral measurement of the CD}

Here due to the size of the beam $(0.8 \mathrm{~cm})$, a series of close lines $(1-\mu \mathrm{m}$ pitch) were written following a Peano function for filling a square of $1 \times 1 \mathrm{~cm}^{2}$. Recall that the quantitative CD in a solid sample that is linearly and circularly birefringent at the same time is not directly measurable. The quantity actually measured by the instrument is the ellipticity of the electromagnetic field (mdeg), which is defined as follows:

$$
\theta=2 \tanh ^{-1}\left(\frac{I_{\mathrm{L}}-I_{\mathrm{R}}}{I_{\mathrm{L}}+I_{\mathrm{R}}}\right)=-\left(\alpha_{\mathrm{R}}-\alpha_{\mathrm{L}}\right) l
$$

The ellipticity is proportional to the $\operatorname{CD} \alpha_{\mathrm{R}}-\alpha_{\mathrm{L}}$, where $\alpha$ is the linear absorption and $l$ is the length of the absorptive layer, only when the sample is isotropic, that is, in samples that do not exhibit either linear birefringence or linear dichroism. Otherwise, it is preferable to use circular anisotropic degree of attenuation (CADA) instead of CD, as it will be called in the following. Moreover, before performing these experiments, we must take into account that the linear and circular properties are now mixed in the same sample. If the circular properties are negligible compared with the linear ones by approximately three orders of magnitude, then they do not perturb the measurement of the linear properties. It is quite the opposite situation when measuring the circular properties. The reverse situation is of course not true, which represents some of the difficulties to overcome. As explained in the experimental details section, when linear and circular properties are not interrelated (that is, separated within the sample depth), the light propagation is no longer reciprocal. Thus, CADA must be measured from both sides of the sample; in addition, its real amplitude cannot be deduced without fitting and without knowing the quantitative values of the linear optical properties, namely, linear dichroism and linear birefringence.

For the data reported in Figure 2, a 160-fs pulse duration laser was used because this pulse duration allows a clear splitting between T1 and T2 thresholds ${ }^{30}$. As shown in Figure 2, below the energy threshold for the appearance of porous nanoplanes and related strong linear birefringence, there is no or negligible CADA. On the contrary, for pulse energy above T2, an ellipticity appears. The sign of the CADA appears to be reversible with laser polarization, that is, positive when the polarization is parallel to the scanning direction and negative when the polarization is perpendicular to the scanning direction; however, it is not generally true, as the linear properties can modify this sign. Notably, the measurements are indeed non-reciprocal, as expected due to the linear optical properties. In Figure 3, we reported samples irradiated in the following conditions: $1030 \mathrm{~nm} 300 \mathrm{fs}, 500 \mathrm{kHz}$, $500 \mu \mathrm{ms}^{-1}$, $0.6 \mathrm{NA}$ and $1 \mu \mathrm{J}$ per pulse (that is, above the T2 threshold). The CADA varies with the direction of the writing 
direction and polarization. The difference from one face to the other face arises from the linear birefringence and its spectral dependence when the linear and circular properties are not located at the same place in depth. The difference reaches a magnitude that is quite high compared with some organic molecules ${ }^{31} . \theta$ can reach $0.1^{\circ}$ in the blue or ultraviolet (UV) range; however, in most samples irradiated under various laser conditions, it is at the level of a few tens of mdeg. This observation should be compared with the linear dichroism that reaches the range of $20-30^{\circ}$ in the same samples. Notice that chiral assemblies of metal nanoparticle devices actually exhibit CD of a few mdeg $^{32}$.

Figure 4 shows that CADA depends on the laser pulse energy as well. At low energy of $\sim 0.2 \mu \mathrm{J}$, CADA does not depend on the sample face order through which the measurement is performed. In other

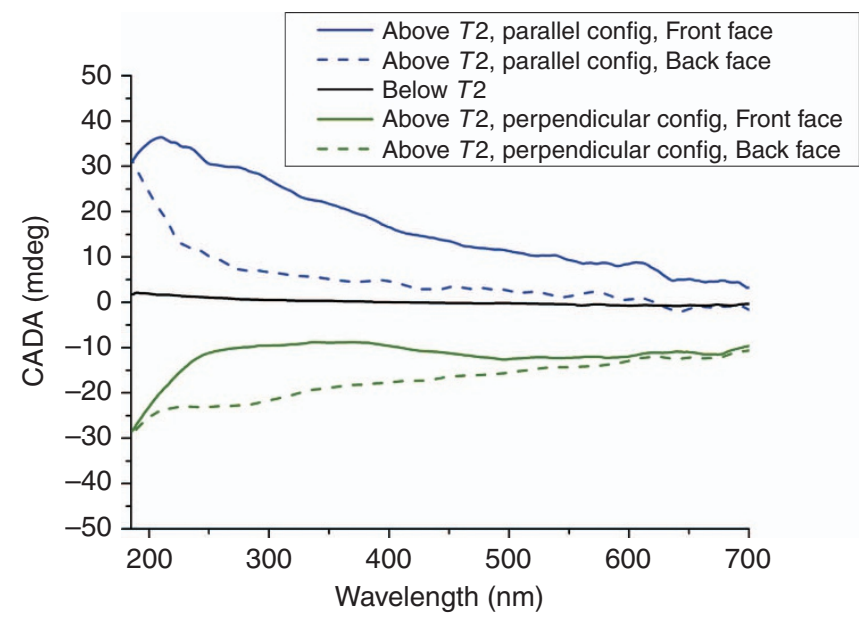

Figure 2 Circular anisotropy degree of attenuation (CADA) according to the probe wavelength, measured below and above the porous nanoplanes energy threshold T2. Laser writing conditions: wavelength: $800 \mathrm{~nm}$, pulse duration: $160 \mathrm{fs}$, repetition rate: $250 \mathrm{kHz}$, numerical aperture: 0.55 , writing speed: $500 \mu \mathrm{m} \mathrm{s}^{-1}$, focal depth: $192 \mu \mathrm{m}$, energy: $0.07 \mu \mathrm{J}$ (below T2) and $1.3 \mu \mathrm{J}$ (above T2).

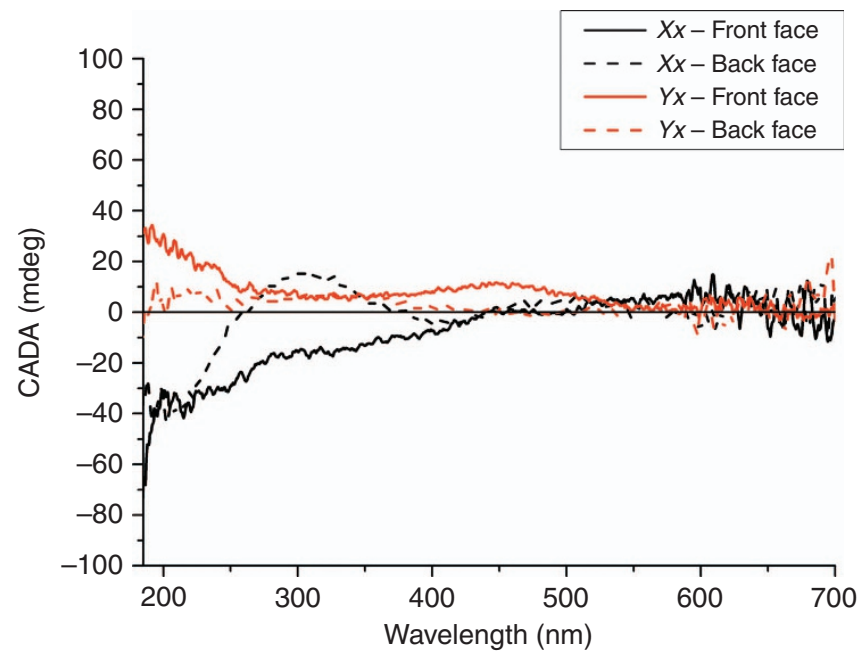

words, linear birefringence is too weak to have a role here if the linear and circular properties are dependent on depth or if the linear and circular properties are not dependent on depth and at the same place. CADA disappears above $1.8 \mu \mathrm{J}$ for the laser conditions used. The scanning electron microscope images show that nanoplanes form clearly at $0.3 \pm 0.1 \mu \mathrm{J}$ and disappear at $1.8 \mu \mathrm{J}$ along with substantial formation of damage. This observation indicates that circular properties are likely appearing in the same conditions as those for nanoplanes formation, that is, the type II regime.

\section{Tentative interpretation}

The measured results above establish that linearly polarized femtosecond laser beam entering an isotropic sample, a glass, under an axially symmetric geometry, is able to break the chiral symmetry of the glass. Notice that because a mirror exists in the middle of the line containing the laser propagation axis and the direction of writing, writing a line breaks axial symmetry but does not break chiral symmetry. This apparently violates the Curie principle resulting in parity violation, as stated in the paper title. Indeed, we discover here a phenomenon in which a material is predicted to be achiral but actually gives rise to a chiral property, for example, optical rotation or CD.

In determining the mechanism of such an unusual process, it must be first mentioned that chiral forces (shearing named as the scissor effect) have been proved to be relevant ${ }^{18,33}$. In addition, linear birefringence axes are expected to be aligned with the written line direction or the laser polarization ones (when it is perpendicular to the written line), which are the apparent symmetry axes of the written object; however, very often it is not the case. This leads to consider that a torque is applied by the light on the matter in the course of the laser direct writing process. Therefore, we are seeking the chiral force exerted on the material that can imprint a twist in the atomic arrangement, it is the case in the crystalline quartz or produce chiral defects from oxygen vacancies, for example. Notice that our observations are not related to the rotation in space of the nanogratings that has been recently reported by Dai et al. ${ }^{34,35}$; indeed, there is no chiral arrangement reported in these two references, as the images are symmetric in a mirror.

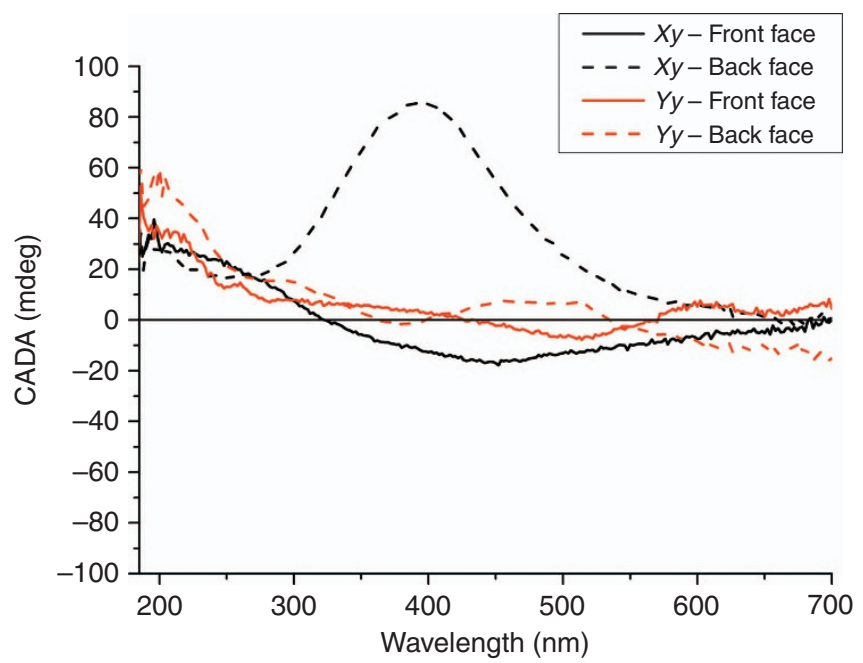

Figure 3 Comparison of spectral circular polarization degree of attenuation (CADA) for four configurations of writing and polarization direction from the two faces of the samples $(X y, Y y, X X$ and $Y X)$. Laser writing conditions: wavelength: $1030 \mathrm{~nm}$, pulse duration: $300 \mathrm{fs}$, repetition rate: $500 \mathrm{kHz}$, numerical aperture (NA): 0.6, energy: $1 \mu \mathrm{J}$ per pulse, writing speed: $500 \mu \mathrm{m} \mathrm{s}^{-1}$, focal depth: $192 \mu \mathrm{m}$. 

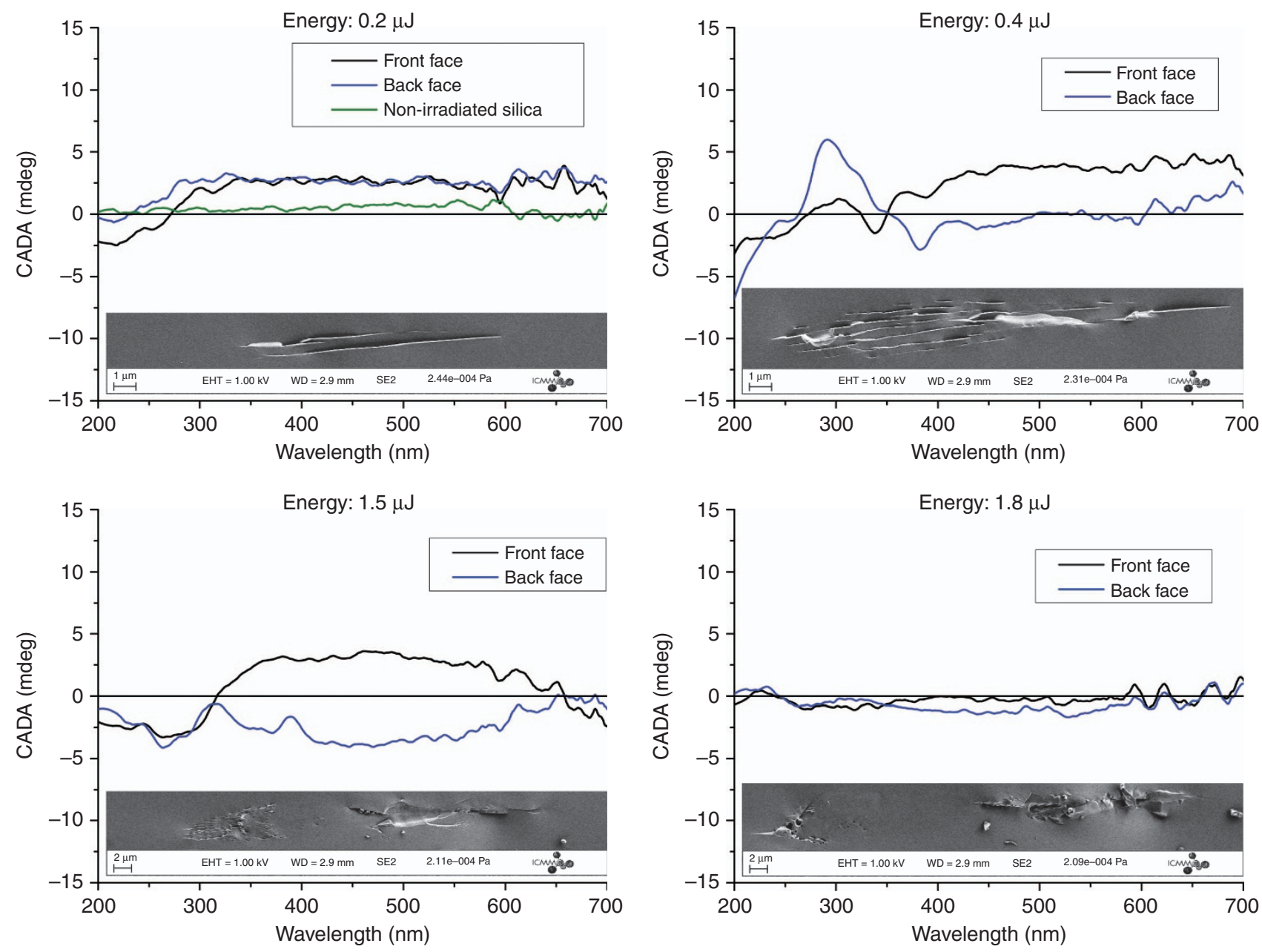

Figure 4 Spectral circular polarization degree of attenuation (CADA) and field emission gun scanning electron microscope secondary electrons images of laser tracks cross-section according to pulse energy for the following laser writing conditions: wavelength: $1030 \mathrm{~nm}$, pulse duration: $300 \mathrm{fs}$, repetition rate: $500 \mathrm{kHz}$, numerical aperture: 0.6 , writing speed: $500 \mu \mathrm{m} \mathrm{s}^{-1}$, focal depth: $192 \mu \mathrm{m}$, configuration is $X x$ (that is, scanning and writing laser polarization horizontal). NB, the laser propagation direction was from the left side to the right side of the picture.

Our experimental results are explained as follows. Intense Gaussian femtosecond laser pulses focused in the material gives rise to multiphoton ionization until saturation in our conditions in the focal area. However, the electron density is not homogeneous due to effect of the ponderomotive force exerted on the free electrons, as it is described by Bethune ${ }^{36}$ and considered again in Ref. 37. The ponderomotive potential $U_{\mathrm{p}}$ is $\sim 10 \mathrm{eV}$ in our conditions ${ }^{38}$. However, notice that the $\mathrm{CD}$ is created by a significant pulse accumulation (1000 pulses per $\mu \mathrm{m})$. Within the approximation of linear polarization, the force is as given in the following Equation (2):

$$
\vec{f}_{\mathrm{fe}}=\left(\vec{P}_{e} \cdot \vec{\nabla}\right) \vec{E}+\frac{\partial \vec{P}_{e}}{\partial t} \Lambda \vec{B}
$$

where $\vec{P}_{e}$ is the polarization of the quasi-free electrons only in the Drude approximation $\left(\vec{P}_{e}=\varepsilon_{0} \chi_{\mathrm{fe}} \vec{E}\right.$ with $\varepsilon_{0} \chi_{\mathrm{fe}}=-\frac{n_{e} e^{2}}{m \omega^{2}}, e$ and $m$ are relative to the quasi-free electrons, and $n_{e}$ is its density). We obtain $\vec{f}_{\mathrm{fe}}=\varepsilon_{0} \chi_{\mathrm{fe}}\left(\frac{\vec{\nabla} \vec{E}^{2}}{2}+\frac{\partial(\vec{E} \Lambda \vec{B})}{\partial t}+\frac{\partial n_{e}}{n_{e} \partial t} \vec{E} \Lambda \vec{B}\right)$. This force pushes the electron from the high-intensity area towards the edge of the laser beam. In the framework of the approximation used here, the force is sensitive to the pulse front tilt. Indeed, it has been shown by several groups that unusual effects, for example, asymmetrical writing of nanostructures or linear birefringence ${ }^{39-41}$, are dependent on the pulse front tilt occurring from the time compression process in the ultrashort pulse laser system. Our laser system exhibits a pulse front tilt oriented $36^{\circ}$ anticlockwise in the $x y$ plane and $0.064^{\circ}$ out of the $z$ axis for a 5 -mm-wide beam before focusing, resulting in an angle of $\sim 60^{\circ}$ from the $z$ axis at the focus. Therefore, the electrons are not only expelled from the center of the beam towards the side but also the pulse front pushes some electrons to only one beam side in the manner of a 'snow-plough', an effect previously described in Ref. 16. The electron density is thus higher on one side of the beam defined by the pulse front tilt only.

However, the experiment shows that the laser polarization has an important role in the orientation of the effect. To achieve such an effect, it is necessary to relax some of the approximations. The first approximation is the linearity of the matter polarization. In the dipolar approximation, the first non-zero term at pump frequency is the third-order polarization; this is the Kerr effect, but this one does not introduce any force component sensitive to laser polarization 
orientation. The next term at the pump frequency is the third-order quadrupolar term, given as follows:

$$
\vec{P}_{\mathrm{NL}}^{3 Q}=\varepsilon_{0} \chi_{\mathrm{eff}, 1}^{(3 q)}|E|^{2} \frac{\partial \vec{B}}{\partial t}+\frac{\varepsilon_{0} \chi_{\mathrm{eff}, 2}^{(3 q)}}{2}(\vec{E} \Lambda \vec{\nabla})|E|^{2}
$$

This term alters the free-electron density according to the laser polarization and gives rise to a non-centrosymmetric density, depending on the pulse front tilt and the laser polarization orientation.

At the end of the pulse, electrons relax and become trapped in the material in $<1$ ps (Ref. 42) resulting in a direct current (DC) field because the counter-ions are much heavier than electrons and are not displaced significantly by the electromagnetic field. There is thus a DC electric field applied in the material in the focal area at the same time as the solid temperature increased due to electron energy loss by electron-phonon coupling during the relaxation process. The solid temperature at this time is high enough for allowing this DC field to distort the ionized atomic network ${ }^{43}$ and, probably, to partly to screen the DC field. A stress field balances the other part. Next, $>1 \mu$ s later, another pulse arrived that has moved slightly. This second pulse changes the charge distribution everywhere except in certain places where the material (especially in the back of the beam displacement) cannot relax because the temperature increase is not high enough and the glass network distortion thus becomes recorded within the medium. Therefore, a stress field remains along the writing trajectory. This gives rise to unusual surface relaxation topography, as has been noted in our previous publications ${ }^{18,33}$.

After the first pulse, the material is under stress that is a non-local property extending largely out of the irradiated area and rendering anisotropic properties onto the matter. As the silica photosensitivity is dependent on the stress field ${ }^{44}$, the coexistence of a DC field and a stress field leads to a possible coupling between them. In such conditions, the material polarization field after pulses must be written as follows:

$$
\vec{P}=\varepsilon_{0} \chi^{0} \vec{E}_{\mathrm{DC}}+\varepsilon_{0} \overline{\overline{\bar{\chi}}} \overline{\bar{\sigma}} \vec{E}_{\mathrm{DC}}
$$

where $\overline{\overline{\bar{\chi}}}$ is the fourth-order piezoelectric tensor and $\overline{\bar{\sigma}}$ is the mechanical stress tensor. In isotropic media, $\overline{\overline{\bar{\chi}}} \overline{\bar{\sigma}}=\chi_{11} \operatorname{Tr}(\overline{\bar{\sigma}}) \overline{\bar{I}}+2 \chi_{12} \overline{\bar{\sigma}}$, where Voigt notation has been used for the susceptibility components, $\operatorname{Tr}(\overline{\bar{\sigma}})$ is the trace of the stress tensor and $I$ is the identity tensor. Thus, the polarization can be written:

$$
\vec{P}=\varepsilon_{0} \chi^{0} \vec{E}_{\mathrm{DC}}+\varepsilon_{0} \chi_{11} \operatorname{Tr}(\overline{\bar{\sigma}}) \overline{\bar{I}} \vec{E}_{\mathrm{DC}}+2 \varepsilon_{0} \chi_{12} \overline{\bar{\sigma}} \vec{E}_{\mathrm{DC}}
$$

The first and second terms in the above expression give rise to the polarization contribution in the same direction as the DC electric field. On the contrary, as the stress tensor cannot in general be reduced to a scalar term, the third term introduces a polarization contribution in a direction that is perpendicular to the DC electric field. The misalignment between $\vec{E}_{\mathrm{DC}}$ and $\vec{P}$ generates a volumic torque, that is, $\vec{P} \Lambda \vec{E}_{\mathrm{DC}}$. However, what are its direction and the sense of rotation? As previously stated, the recorded DC field is oriented in the direction of the pulse front tilt (here $36^{\circ}$ anticlockwise in the $x, y$ plane) deviated by the action of the laser polarization. In addition, the stress field deduced from the surface topography analysis reveals a shear stress that can be written as below in the written line reference containing



Figure 5 Scheme showing the combined action of $E_{D C}$ and the stress field giving rise to a volumic torque at the end of the written line. $\vec{P}_{\perp}$ is the component of $\vec{P}$ perpendicular to $\vec{E}_{\mathrm{DC}}$.

the light propagation direction and the movement of the beam $(\vec{v}, \vec{k} \Lambda \vec{v}, \vec{k})$, as described in Figure 5:

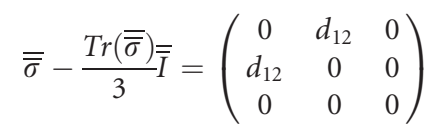

This volumic torque may be responsible for the chiral atomic arrangements thought to underlie the present observations by cumulative effect. Thus, the control of the pulse front tilt or of a simple intensity gradient ${ }^{45}$ and of the laser polarization according to the displacement should lead to the control of circular properties in a matter (pure silica glass here but in principle in any glasses), where such effect is not currently possible before irradiation. Femtosecond direct laser writing conjugated to pulse front tilt engineering is thus a new functionalization process of the glass that may be further extended to other optical materials.

\section{CONCLUSIONS}

We showed that the irradiation of an achiral material (pure silica glass) with achiral light (linear polarized Gaussian beam) from a femtosecond laser system can render the material chiral. However, the pulse energy must overcome the energy threshold related to the production of nanogratings giving rise to form birefringence. The circular birefringence (optical rotation) appears to be $\sim 5^{\circ} \mathrm{mm}^{-1}$, which is adequate for practical applications. A significant $\mathrm{CD}$ also appears that is close to the value found for organic molecules; this result enables consideration of devices based on chiral assemblies of metal nanoparticles. This last result is surprising because pure quartz exhibits no $\mathrm{CD}$ in the UV-visible range. Moreover, from the theory developed above, we propose that the breaking of symmetry arises from the chirality of the set, including the DC electric field (defined by the pulse front tilt and the laser polarization), the direction of light propagation and the direction of scanning.

This new functionalization process could provide a platform for a versatile new class of photonic devices. With regard to biomimetics, we can imagine production of a cholesteric liquid crystal analogous optical devices using tiny lengths of inorganic glass, that is, 'twisted silica glass' rather than a macroscopic assembly ${ }^{14}$. An example could be the production of 'chiral fiber gratings' that can be harnessed for producing sensors, polarizers, optical isolators and filter/lasers. Femtosecond laser processing thus offers a new advantage, partly in a nonconventional way: it allows restructuring of our most important optical material to enable chirality and rotating power. The important scientific point is that the pulse duration is so short that direct action on the lattice is not possible. However, the creation of the electric field creates a memory effect staying between pulses that may act on the material. Other experiments are planned to study the level of CD according to the pulse number and its spatial distribution along the laser traces. Last, we think that the use of high peak power and 
ultrashort pulses is a powerful tool for the development of new applications in materials science for optics.

\section{CONFLICT OF INTEREST}

The authors declare no conflict of interest.

\section{ACKNOWLEDGEMENTS}

This work has been performed in the framework of the FLAG (Femtosecond Laser Application in Glasses) international project with the support of FP7PEOPLE-IRSES e-FLAG 247635 from the Agence Nationale pour la Recherche (ANR-09-BLAN-0172-01).

1 Davis KM, Miura K, Sugimoto N, Hirao K. Writing waveguides in glass with a femtosecond laser. Opt Lett 1996; 21: 1729-1731.

2 Shimotsuma Y, Kazansky PG, Qiu JR, Hirao K. Self-organized nanogratings in glass irradiated by ultrashort light pulses. Phys Rev Lett 2003; 91: 247405.

3 Schaffer CB, Brodeur A, Nishimura N, Mazur E. Laser-induced microexplosions in transparent materials: microstructuring with nanojoules. Proc SPIE 1999; 3616: 143.

4 He L, Sheehy K, Culbertson W. Femtosecond laser-assisted cataract surgery. Curr Opin Ophthalmol 2011; 22: 43-52.

5 Glezer EN, Milosavljevic M, Huang L, Finlay RJ, Her TH et al. Three-dimensional optical storage inside transparent materials. Opt Lett 1996; 21: 2023-2025.

6 Podlipensky A, Abdolvand A, Seifert G, Graener H. Femtosecond laser assisted production of dichroitic 3D structures in composite glass containing Ag nanoparticles. Appl Phys A Mater Sci Process 2005; 80: 1647-1652.

7 Watanabe M, Sun HB, Juodkazis S, Takahashi T, Matsuo S et al. Three-dimensional optical data storage in vitreous silica. Jpn J Appl Phys 1998; 37: L1527-L1530.

8 Monat C, Domachuk P, Eggleton BJ. Integrated optofluidics: a new river of light. Nat Photonics 2007; 1: 106-114.

9 Bellouard Y, Said A, Dugan M, Bado P. Fabrication of high-aspect ratio, micro-fluidic channels and tunnels using femtosecond laser pulses and chemical etching. Opt Express 2004; 12: 2120-2129.

10 Corrielli G, Crespi A, Geremia R, Ramponi R, Sansoni L et al. Rotated waveplates in integrated waveguide optics. Nat Commun 2014; 5: 4249.

11 Beresna M, Gecevičius M, Kazansky PG. Polarization sensitive elements fabricated by femtosecond laser nanostructuring of glass. Opt Mater Express 2011; 1: 783-795.

12 Fernandes LA, Grenier JR, Herman PR, Aitchison JS, Marques PVS. Stress induced birefringence tuning in femtosecond laser fabricated waveguides in fused silica. Opt Express 2012; 20: 24103-24114.

13 Heilmann R, Gräfe M, Nolte S, Szameit A. Arbitrary photonic wave plate operations on chip: realizing Hadamard, Pauli-X, and rotation gates for polarisation qubits. Sci Rep 2014; 4: 4118.

14 Desmarchelier R, Lancry M, Gecevicius M, Beresna M, Kazansky PG et al. Achromatic polarization rotator imprinted by ultrafast laser nanostructuring in glass. Appl Phys Lett 2015; 107: 181111.

15 Sudrie L, Franco M, Prade B, Mysyrowicz A. Writing of permanent birefringent microlayers in bulk fused silica with femtosecond laser pulses. Opt Commun 1999; 171: 279-284.

16 Kazansky PG, Yang WJ, Bricchi E, Bovatsek J, Arai A et al. "Quill" writing with ultrashort light pulses in transparent materials. Appl Phys Lett 2007; 90: 151120.

17 Lancry M, Poumellec B, Canning J, Cook K, Poulin JC. Ultrafast nanoporous silica formation driven by femtosecond laser irradiation. Laser Photonics Rev 2013; 7 953-962.

18 Poumellec B, Sudrie L, Franco M, Prade B, Mysyrowicz A. Femtosecond laser irradiation stress induced in pure silica. Opt Express 2003; 11: 1070-1079.

19 Lancry M, Poumellec B, Desmarchelier R, Bourguignon B. Oriented creation of anisotropic defects by IR femtosecond laser scanning in silica. Opt Mater Express 2012; 2: 1809-1821.

20 Poumellec B, Lancry M, Desmarchelier R, Hervé E, Brisset F et al. Asymmetric Orientational Writing in glass with femtosecond laser irradiation. Opt Mater Express 2013; 3: 1586-1599.

21 Curie $P$. Sur la symétrie dans les phénomenes physiques, symétrie d'un champ électrique et d'un champ magnétique. Ann Fond Louis Broglie 1994; 19: 137.
22 Österberg U, Margulis W. Dye laser pumped by Nd: YAG laser pulses frequency doubled in a glass optical fiber. Opt Lett 1986; 11: 516-518.

23 Noorduin WL, Bode AAC, van der Meijden M, Meekes H, van Etteger AF et al. Complete chiral symmetry breaking of an amino acid derivative directed by circularly polarized light. Nat Chem 2009; 1: 729-732.

24 Desmarchelier R, Poumellec B, Brisset F, Mazerat S, Lancry M. In the heart of femtosecond laser induced nanogratings: from porous nanoplanes to form birefringence. World J Nano Sci Eng 2015; 5: 115-125.

25 Dekker P, Ams M, Marshall GD, Little DJ, Withford MJ. Annealing dynamics of waveguide Bragg gratings: evidence of femtosecond laser induced colour centres. Opt Express 2010; 18: 3274-3283.

26 Diels JC, Rudolph W. Ultrashort Laser Pulse Phenomena. New York: Academic Press. 1996.

27 Bricchi E, Klappauf BG, Kazansky PG. Form birefringence and negative index change created by femtosecond direct writing in transparent materials. Opt Lett 2004; 29: 119-121.

28 Chandrasekhar S. The optical rotatory power of Quartz and its variation with temperature. Proc Ind Acad Sci 1952; 35: 103-113.

29 Kleinman DA, Spitzer WG. Theory of the optical properties of quartz in the infrared. Phys Rev 1962; 125: 16-30.

30 Poumellec B, Lancry M, Chahid-Erraji A, Kazansky PG. Modification thresholds in femtosecond laser processing of pure silica: review of dependencies on laser parameters. Opt Mater Express 2011; 1: 766-782.

31 Hnatovsky C, Taylor RS, Simova E, Rajeev PP, Rayner DM et al. Fabrication of microchannels in glass using focused femtosecond laser radiation and selective chemical etching. Appl Phys A 2006; 84: 47-61.

32 Schreiber R, Luong N, Fan ZY, Kuzyk A, Nickels PC et al. Chiral plasmonic DNA nanostructures with switchable circular dichroism. Nat Commun 2013; 4: 2948

33 Poumellec B, Lancry M, Poulin JC, Ani-Joseph S. Non reciprocal writing and chirality in femtosecond laser irradiated silica. Opt Express 2008; 16: 18354-18361.

34 Dai $Y$, Wu GR, Lin X, Ma GH, Qiu JR. Femtosecond laser induced rotated 3D self-organized nanograting in fused silica. Opt Express 2012; 20: 18072-18078.

35 Dai Y, Ye JY, Gong M, Ye XY, Yan XN et al. Forced rotation of nanograting in glass by pulse-front tilted femtosecond laser direct writing. Opt Express 2014; 22 $28500-28505$.

36 Bethune DS. Optical second-harmonic generation in atomic vapors with focused beams Phys Rev A 1981; 23: 3139-3151.

37 Gecevičius M, Beresna M, Zhang JY, Yang WJ, Takebe $\mathrm{H}$ et al. Extraordinary anisotropy of ultrafast laser writing in glass. Opt Express 2013; 21: 3959-3968.

38 Couairon A, Mysyrowicz A. Femtosecond filamentation in transparent media. Phys Rep 2007: 441: 47-189.

39 Bellouard Y, Hongler MO. Femtosecond-laser generation of self-organized bubble patterns in fused silica. Opt Express 2011; 19: 6807-6821.

40 O'shea P, Kimmel M, Gu X, Trebino R. Highly simplified device for ultrashort-pulse measurement. Opt Lett 2001; 26: 932-934.

41 Yang WJ, Kazansky PG, Shimotsuma Y, Sakakura M, Miura K et al. Ultrashort-pulse laser calligraphy. Appl Phys Lett 2008; 93: 171109.

42 Lancry M, Groothoff N, Poumellec B, Guizard S, Fedorov N et al. Time-resolved plasma measurements in Ge-doped silica exposed to infrared femtosecond laser. Phys Rev B 2011; 84: 245103.

43 Lancry M, Poumellec B, Guizard S. Comparison between plasma properties and damage thresholds in doped silica exposed to IR temtosecond laser. J Laser Micro Nanoeng 2012; 7: 217-225.

44 Shimotsuma Y, Hirao K, Qiu JR, Miura K. Nanofabrication in transparent materials with a femtosecond pulse laser. J Non-Cryst Solids 2006; 352: 646-656.

45 Salter PS, Booth MJ. Dynamic control of directional asymmetry observed in ultrafast laser direct writing. App/ Phys Lett 2012; 101: 141109.

(1) (2) This work is licensed under a Creative Commons AttributionSA Nommercial-ShareAlike 4.0 International License. The images or other third party material in this article are included in the article's Creative Commons license, unless indicated otherwise in the credit line; if the material is not included under the Creative Commons license, users will need to obtain permission from the license holder to reproduce the material. To view a copy of this license, visit http:// creativecommons.org/licenses/by-nc-sa/4.0/

(C) The Author(s) 2016 